\title{
Téoros
}

Revue de recherche en tourisme

\section{Information et tourisme : un alliage difficile ?}

\section{Normand Cazelais}

Volume 4, numéro 1, mars 1985

\section{L'information touristique}

URI : https://id.erudit.org/iderudit/1080734ar

DOI : https://doi.org/10.7202/1080734ar

Aller au sommaire du numéro

\section{Éditeur(s)}

Université du Québec à Montréal

\section{ISSN}

0712-8657 (imprimé)

1923-2705 (numérique)

Découvrir la revue

\section{Citer cet article}

Cazelais, N. (1985). Information et tourisme : un alliage difficile ? Téoros, 4(1), 2-3. https://doi.org/10.7202/1080734ar d'utilisation que vous pouvez consulter en ligne.

https://apropos.erudit.org/fr/usagers/politique-dutilisation/ 


\title{
Information et tourisme: un alliage difficile?
}

\author{
par Normand Cazelais*
}

\section{Une information spécialisée}

L'information touristique est une information spécialisée. Même si une bonne partie de son contenu - de ses nouvelles, en fait - est incorporée, au fil des jours, à l'information générale ou est traitée comme un matériau relevant des transports, de la vie économique ou politique, du monde des lettres ou même des beaux-arts! Ainsi en est-ill des investissements que des stations de ski consentiront dans la fabrication de la neige artificielle, des retombées de tout ordre des théatres d'été dans leurs régions d'accueil, de l'écrasement d'un avion dans une ille des Antilles ou des malheurs d'un groupe de voyageurs retenus à Hong-Kong ou ailleurs gråce aux bons soins d'un intermédiaire peu consciencieux.

Le champ d'intérêt de l'information touristique, à l'image de son objet, est vaste, très vaste. Cependant, dans l'esprit d'un grand nombre de lecteurs et de responsables de salles de rédaction, I'information touristique se résume - sinon se réduit - à la production de topos vantant -nécessairementles merrites de destinations le plus souvent exotiques, éloignées ou carrément inaccesibles, chères, porteuses de rêve, d'évasion ou de frustration... D'où la réputation douteuse de l'information touristique et de ses agents.

Les voies que prend d'autre part l'incitation au voyage sont, quant a elles, fort variées sans qu'on pense, dans la plupart des cas, leur accoler les épithètes du paragraphe précédent. Ainsi, qui n'a vu les images ouatees et lumineuses du film d'Yves Boisset, Le taxi mauve, et n'a eu le goût impératif de partir pour l'Irlande? Qui $n^{r} a$ écouté Desafinado et n'a senti monter en lui le désir de connaitre le Brésil? Qui n'a lu Hemingway ou Durrell et n'a voulu visiter l'Espagne ou l'Égypte? Qui n'a dévoré un reportage sur le miracle économique japonais sans mettre au rang de ses secrètes ambitions l'intention de voir sur place

"Normand Cazelais est géographe, chroniqueur en tourisme à la pige et chargé de cours en tourismę a r Universite de Montréal (certificat en loisir). le soleil se lever en ce pays? Même les atrocités des guerres, des famines, des cataclysmes donnent le goùt de partir car elles livrent, elles aussi, par un ensemble d'images frappantes, une vision de l'ailleurs. D'où l'ambiguité qui teinte l'information touristique... car, après tout, dit-on, n'importe qui peut en faire: il ne s'agit pas d'être spécialiste pour cela, il suffit d'aimer voyager.

Résultat, même si on parle abondamment de tourisme ou de ses multiples manifestations et expressions dans les médias écrits ou électroniques, qu'ils soient hebdomadaires, mensuels ou trimestriels, mẹme si quelques grands et rares quotidiens ont leurs journalistes attitrés, il y a - au Québec, à tout le moins - très peu d'informateurs spécialisés en tourisme, comme si l'information touristique, en tant qu'information spécialisée, n'existait pas... Pourtant, elle existe, pourrait-on dire à l'exemple de Galilée..

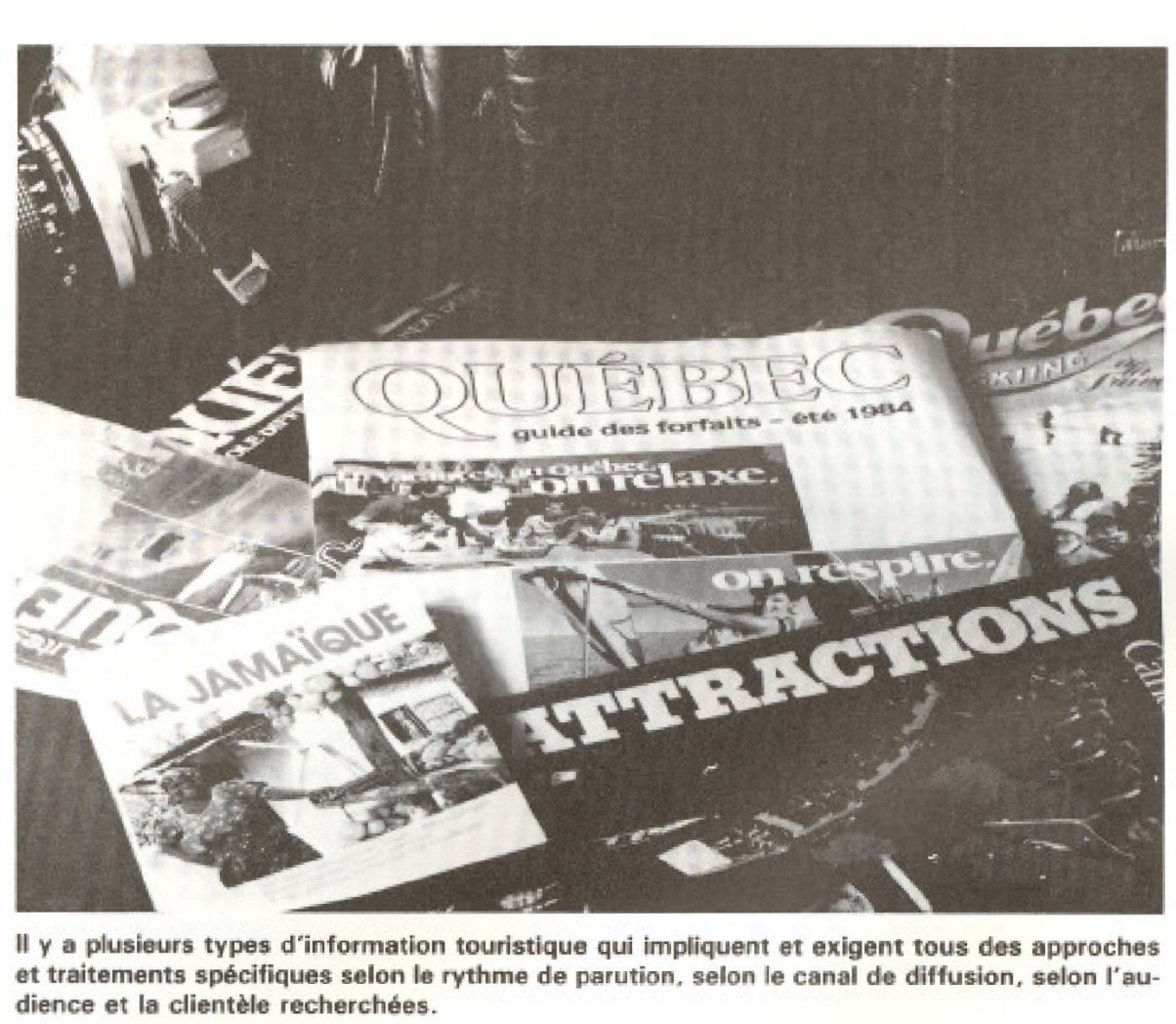

II $\curlyvee$ a plusieurs types d'information touristique qui impliquent et exigent tous des approches et traitements specifiques selon le rythme de parution. selon le canal de diffusion, selon I'audience et la clientèle recherchées.

\section{Des caractères particuliers}

Pourtant, comme dans toutes les autres informations spécialisées, il y a plusieurs types d'information touristique qui impliquent et exigent tous des approches et traitements spécifiques selon le rythme de parution, selon le canal de diffusion, selon I'audience et la clientẻle recherchées, et caetera. L'information touristique d'un quotidien ne sera pas celle d'un magazine ni celle d'une émission de télé. L'information destinée au grand public ne ressemble pas à celle, dite du "trade", qui s'adresse au professionnels d'un secteur d'activités en particulier, comme celles-ci se distinguent de l'information scientifique et statistique, de l'information corporative produite par les entreprises commerciales, les gouvernements et leurs organismes, comme toutes celles-lá sont differrentes de l'information véhiculée dans les guides écrits, comme il y a autant de catégories de guides qu'il y a de collections et de mai- 


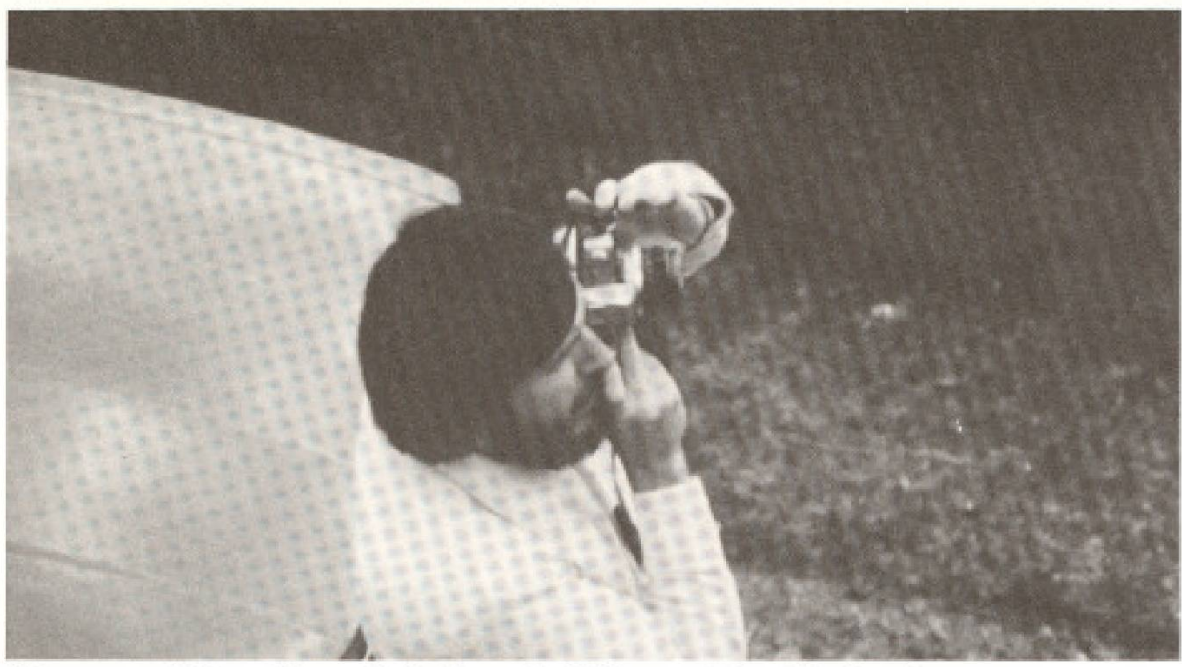

"En un mot, faire vraiment de I'information!" Normand Cazelais chroniqueur... et photographe.

sons d'édition, comme il y a une mer, un océan de différence entre l'information fournie dans un livre et celle transmise oralement par un guide, fut-ill düment reconnu. Avec ses caractéristiques propres, l'information touristique participe done aux imperratifs et aux problèmes de l'information tout court

Mais poursuivons l'analyse de ses caractéristiques, notamment de celle destinée au public en général. C'est une information, quand elle traite de destinations, qui est décalée, déphasée dans le temps, à la fois projective et rétrospective, quí regarde dans un meme mouvement loin devant elle et derrière son épaule. En effet, sauf pour des topos ou des reportages pour les medias électroniques et les quotidiens sur des excursions d'un ou de quelques jours dans un lieu ou une région pas trop éloignés, l'information touristique parle d'une réalité à venir, d'une réalité anticipée; un article écrit en mai ou juin sur le ski dans les Alpes (praticable de décembre à avril) paraïtra en septembre-octobre, au moment oú les gens commencent pour la plupart à planifier leur prochain voyage d'hiver, au moment aussi où transporteurs et grossistes commencent a les allecher $\mathrm{a}$ grands renforts de publicité

Cette information anticipée * et c'est là le paradoxe - s'appule sur le passé sinon sur un acte de foi. Rares - c'est un euphémisme - sont le reportages qui s'appuient sur un vovage récent, tout "chaud"; leur élaboration ou leur rédaction a pu se faire immédiatement au retour mais leur parution ne se fait que plusieurs semaines ou mois plus tard, pour coller précisément á l'actualité touristique anticipée, déphasée. Un informateur touristique ne voyage pas en haute-saison car il serait trop tard, les gens sont déjà rendus à destination ou ont déjả fait leur choix. Reprenons notre exemple: I'expérience qui a permis la rédaction de l'article en mai-juin s'est déroulée au cours de l'hiver précédent. Les variantes sont nombreuses mais le trait fondamental demeure: un "papier" paru à la fin juin sur la Gaspésie dans un journal s'alimentera à un voyage fait en mail ou au cours d'années antérieures, donc à une "autre" Gaspésie. D'oủ l'acte de foi, celui de croire à l'instant où l'information est transmise que la destination et les conditions d'exercice des activités décrites n'auront pas changé. Ou, du moins, pas trop.

Autre aspect, en partie corollaire au précédent: les clients désireux de partir en voyage achètent des destinations et des produits qu'ils ne peuvent tester . éprouver avant de partir, c'est-â-dire avant de les consommer. Et, mème s'ils les connaissent d'expérience, pour y être allés une ou plusieurs fois, ils ne peuvent contröler des agents externes comme les conditions climatiques, des troubles politiques, des grèves, des fluctuations économiques, des cataclysmes naturels qui, eux, peuvent profondément transformer lesdits produits et destinations. Ils ne peuvent le faire parce que l'objet de leur consommation est ailleurs, est l'ailleurs. D'où l'importance de l'information en ce domaine.

Nouveau paradoxe, I'information touristique, tant par beaucoup de joumalistes que par nombre de responsables de rédaction, $\pi$ 'est pas tellement prise au sérieux. C'est ce qui explique que des médias électroniques, des magazines, des hebdomadaires et même des quotidiens ne paient pas - ou si peu! - les textes de vovage des pigistes qu'ils utilisent: après tout, se disent-ils, le voyage lqui a été offert par la destination, un transporteur, un grossiste ou un amalgame de tous ces intervenants) n'est-il pas en lui-même une récompense, un salaire? Dangereux et triste raisonnement, n'est-ce pas, surtout quand il s'agit d'information? Ce qui explique également que bonne quantité de magazines - même parmi les plus respectés - n'ont pas de collaborateurs spécifiquement affectés à la chronique touristique, contrairement aux chroniques de livres, de disques, de politique, de jardinage, d'automobile, etc. Deux raisons interviennent alors: tout le monde peut parler de voyage - "Y a rien làll" - et le désir de réserver aux responsables et aux employés du magazine les voyages de presse que l' "industrie" propose régulièrement chaque année.

\section{La question de l'éthique}

Nous revenons au point de départ. $\mathrm{C}^{\prime}$ est en effet l'objet spécifique de l'information touristique, â savoir l'ailleurs, qui pose problème et qui déforme la perspective, la façon de considérer et de traiter cette information spécialisée. D'où le peu de sérieux qu'on lui accorde de l'extérieur parce que, voyager, c'est un privilège sinon un salaire en soi et parce que, conséquemment, l'objectivité sinon l'éthique y sont pratiquement impossibles.

Qu'on se leurre pas: toutes ces considérations réfèrent, au-delà de la question touristique, aux conditions mèmes d'exercice de l'information en soi. Tout joumaliste, tout chroniqueur, tout informateur a pour tâche, quand il fait son métier, d'aller à la recherche de donnees et de renseignements, de les digérer, de les ordonner et de les reformuler avec ce moven particulier qu'est son reportage, son analyse, son topo. Non seulement va-t-ill chercher l'information, ill est en outre la cible de sollicitations répétées de la part d'individus, d'organismes et d'entreprises de toute nature: communiquess, conferences de presse et autres moyens d'information corporative, brochures publicitaires, invitations, dīners d'affaires, lobbys politiques, commerciaux, professionnels et j'en passe.

II $n^{\prime} y$ a pas que les sociétés aériennes ou les destinations touristiques qui soignent leur image et qui tentent, dans leur action quotidienne, d'utiliser les médiás d'information pour ce faire: distributeurs de films, maisons d'édition, clubs sportifs, fabricants de contre-plaqués, de bas de nylon ou de cosmétiques, banques, partis politiques, tous cherchent à s'en servir pour attirer l'attention, pour faire la promotion de leurs idées et de leurs produits. Et partout, dans le secteur de l'information touristique comme dans les autres, la distinction entre l'information et la publicité - déguisée ou non - est ténue mais claire: c'est la compétence et la conscience professionnelle de la personne qui exerce ce métier qui lui permettront de préserver sa liberté d'action, son autonomie et de garder à la fois le respect de lui-meme, de ses informateurs et du public. En un mot, de faire vraiment de l'information.

Dans le domaine de l'information touristique, les pressions de la promotion, le "glamour" des "objets d'intérêt" et les structures des canaux de diffusion sont tels que les problèmes d'éthique et de liberté d'expression $y$ sont certes plus aigus. 